\title{
Leishmaniose visceral infantil: relato de caso
}

\author{
Infantile visceral leishmaniasis: case report
}

\section{Luilson Geraldo Coelho Júnior ${ }^{1}$, Alessandra de Paiva Wanderley ${ }^{1}$, Maire Stefani Lemes ${ }^{1}$, Bárbara Cristina dos Santos Ribeiro Leite ${ }^{1}$, Barbara Keroleny Viana Cabrobó1, Gabrielly Borges Machado², Maria Luíza Gonçalves dos Reis Monteiro ${ }^{3}$}

Coelho Junior LG, Wanderley AP, Lemes MS, Leite BCSR, Cabrobó BKV, Machado GB, Monteiro MLGR. Leishmaniose visceral infantil: relato de caso / Infantile visceral leishmaniasis: case report. Rev Med (São Paulo). 2016 jul.-set.;95(3):133-7.

RESUMO: A leishmaniose visceral (LV) é uma infecção causada por protozoários tripanosomatídeos do gênero Leishmania, as espécies são: Leishmania donovani e Leishmania infantum (conhecido como Leishmania chagasi na América do Sul). A LV é uma doença parasitária grave, com elevada morbimortalidade em todo o mundo. É considerada a doença tropical mais negligenciada, em termos de desenvolvimento de novos medicamentos. Considerada um importante problema de saúde em todo o Brasil, principalmente na área endêmica de ParacatuMG. Acomete principalmente crianças com menos de cinco anos de idade. A transmissão ocorre através da picada de fêmeas hematófagas dos vetores Lutzomyia longipalpis ou L. cruzi, infectados pela Leishmania (L.) chagasi. Os sinais clínicos da LV variam de acordo com a resposta imune do hospedeiro, podendo assumir formas assintomáticas, oligossintomáticas e a forma clássica; os principais sinais incluem febre, hepatoesplenomegalia, perda de peso e pancitopenia. Indivíduos não tratados, crianças desnutridas, indivíduos infectados pelo HIV ou em uso de drogas imunossupressoras são grupos de risco, sendo que nesses casos a LV apresenta alta letalidade. O diagnóstico é feito através da demonstração da Leishmania em aspirados de linfonodo, medula óssea e baço; por meio de sorologia, ou teste rápido como o rK39, são amplamente utilizados, e apresentam sensibilidade variável. $\mathrm{O}$ tratamento é realizado com antimoniais pentavalentes e anfotericina B lipossomal ou desoxicolato. Não há vacina humana; portanto o combate ao vetor é a melhor medida de prevenção. Relatamos um caso de LV em criança de 04 anos de idade, natural e procedente de Paracatu-MG.

Descritores: Leishmania infantum; Leishmaniose visceral; Crianças; Prognóstico.

ABSTRACT: Visceral leishmaniasis (VL) is an infection caused by protozoa of the genus Leishmania tripanosomatídeos, the species are: Leishmania donovani and Leishmania infantum (known as Leishmania chagasi in South America). The VL is a severe parasitic disease with high morbidity and mortality worldwide. It is considered the most neglected tropical disease, in terms of the development of new medicines. Considered an important health problem throughout Brazil, mainly in the endemic area of Paracatu, MG. It affects mainly children under five years of age. Transmission occurs through the bite of female Lutzomyia longipalpis blood-feeding of vectors or L. cruzi infected by Leishmania (L.) chagasi. The clinical signs of VL vary according to the immune response of the host, and may take forms asymptomatic, oligosymptomatic metastases and the classical form; the main signs include fever, hepatosplenomegaly, weight loss and pancytopenia. Untreated individuals, malnourished children, individuals infected by HIV or in use of immunosuppressant drugs are risk groups, being

1. Acadêmico de Medicina, Faculdade Atenas, Paracatu -MG, Brasil.E-mail: luilson-junior@hotmail.com, tifa.valentine@gmail.com, mairi-stefani@hotmail.com, barbaracleite@yahoo.com, barbarakeroleny@gmail.com.

2. Médica Infectologista, Faculdade Atenas, Paracatu - MG, Brasil. E-mail: Gabyborges100@hotmail.com.

3: Médica Patologista, Departamento de Patologia Geral, Universidade Federal do Triângulo Mineiro, Uberaba - MG, Brasil. E-mail: marialuizapatologia@gmail.com

Autor para correspondência: Luilson Geraldo Coelho Júnior. Rua Bernardo Caparucho, 30, apart. 04 - Bela Vista. Paracatu, MG, Brasil.38600-000. E-mail: luilson-junior@hotmail.com 
that in these cases the VL presents high lethality. The diagnosis is made through demonstration of Leishmania in lymph node aspirates, bone marrow and spleen; through serology, or rapid test as the rK39, are widely used and present variable sensitivity. The treatment is performed with antimony pentavalents and liposomal amphotericin B or desoxycholate. There is no human

\section{INTRODUÇÃO}

A leishmaniose visceral (LV) é uma doença tropical negligenciada; vista como uma das seis doenças endêmicas consideradas como principais problemas de saúde pública em todo mundo. Apresenta incidência global estimada de 500.000 novos casos por ano e mais de 50.000 mortes anuais ${ }^{1,2}$. A doença ocorre em 65 países, o maior número de casos da doença, cerca de $90 \%$, foi notificada em Bangladesh, Brasil, Etiópia, Índia, Nepal e Sudão².

Mesmo com as estratégias de controle da doença propostas pelo Ministério da Saúde, o Brasil apresenta aumento progressivo do número de casos de LV. Entre 1999 e 2008, o número médio anual de casos foi de 3379 , e a incidência foi de 1,9 casos por 100.000 habitantes. Houve um aumento considerável na taxa de letalidade da LV, em 1999 a taxa era de 3,4\%, em 2008 o valor foi para 5,5\%, correspondendo a um aumento de $61,8 \% \%^{1,3,4}$.

A LV, também conhecida como calazar, é uma doença infecciosa, não contagiosa, de caráter sistêmico. Causada por um protozoário intracelular obrigatório, do gênero Leishmania. É transmitida através da picada das fêmeas hematófagas de flebotomíneos. A infecção causada pela Leishmania pode causar um vasto espectro de manifestações clínicas, sendo que a LV é a forma mais grave e quase sempre letal, se o tratamento não é adequado e não é instituído de maneira precoces.

A espécie causadora de LV é a Leishmania donovani na Ásia e África Ocidental; já a Leishmania infantum é a espécie circulante, causadora de LV na Europa, Norte da África e América Latina. A distribuição geográfica da LV, na maioria das vezes, está limitada a focos infecciosos endêmicos em regiões bem identificadas; todavia, observase que a LV emergiu em epidemias mundiais, além de ser considerada uma das infecções oportunistas nos indivíduos infectados pelo vírus da imunodeficiência humana ${ }^{6}$.

Amaioria dos casos de LV são infecção assintomática, ou oligossintomática, cujos sintomas são moderados ou transitórios, sendo caracterizado por diarréia, tosse seca, adinamia, febrícula, sudorese e hepatoesplenomegalia discreta; podendo ou não evoluir para forma clássica da doença. O calazar clássico é caracterizado por febre, hepaotesplenomegalia, com esplenomegalia pronunciada e volumose; perda de peso, distensão abdominal; na forma tardia da doença, os pacientes podem apresentar edema e $\operatorname{ascite}^{7,8,9}$. vaccine; therefore the fight against vector is the best preventive measure. There is no human vaccine; therefore the fight against vector is the best preventive measure. We report one case of VL in children 04 years of age, natural and coming in Paracatu, MG.

KEY WORDS: Leishmania infantum; Visceral leishmaniasis; Children; Prognosis.

O diagnóstico da doença é baseado no encontro do parasita no tecido da medula óssea, baço, fígado ou linfonodos. Testes sorológicos como imunofluorescência indireta, ELISA, são utilizados para diagnosticar a doença; além disso, técnicas de biologia molecular, reação em cadeia da polimerase, podem ser realizadas. Todavia, os testes apresentam sensibilidade, especificidade variáveis entre si, além disso, o custo e disponibilidade destes testes devem ser analisados na prática clínica. Quando não é possível o diagnóstico laboratorial, o início do tratamento é baseado nos achados clínico-epidemiológicos ${ }^{3,4,7}$.

As classes de drogas utilizadas para o tratamento de LV no Brasil são restritas. No final da década de 40, o antimonial pentavalente foi introduzido no Brasil, para tratamento da LV. Todavia, nos anos 1980 introduziram a anfotericina B desoxicolato, seguido de formulações lipídicas como a anfotericina B lipossomal, esta última, apresenta alta eficácia e baixa toxicidade ${ }^{10,11}$.

As diferenças observadas na eficácia clínica das drogas dependem de vários fatores, tais como, área geográfica de infecção, desenvolvimento de resistência, coinfecção LV-HIV, desnutrição. Estas condições levam ao aumento de resistência à ação das drogas, maior tempo de hospitalização e maior custo do tratamento ${ }^{10,12,13}$.

A LV no Brasil acomete principalmente a população pediátrica; sabe-se que uma característica importante da LV é que, quanto maior a incidência da doença, maior o risco para as crianças mais jovens. A imunidade se desenvolve com a idade, assim sendo, a incidência da doença e do óbito em crianças menores, depende da maior suscetibilidade à infecção e da imunodepressão observada ${ }^{7}$.

Relatamos um caso de LV em criança, pré-escolar, procedente de área endêmica no Noroeste Minas Gerais, Paracatu-MG.

\section{RELATO DE CASO}

Pré-escolar, quatro anos de idade, sexo feminino, parda, natural e procedente de Paracatu-MG, reside na área urbana da cidade de Paracatu - MG.

Cartão vacinal incompleto não foi realizado aos quatro anos o reforço da DTP e VOP. Internação prévia devido a acidente automobilístico. Apresenta condições precárias de moradia, sem saneamento básico. Possui dois cachorros de estimação. 
Deu entrada no setor de emergência do Hospital Municipal de Paracatu em março de 2016 apresentando febre de $39^{\circ} \mathrm{C}$ persistente há 02 dias, sem outras queixas. Os genitores da criança revelaram que foi realizada sorologia para LV nos cães, com resultado não reagente.

Paciente em regular estado geral, prostrada, hipocorada 2+/4, hidratada. Sinais vitais: Fr: 28irpm, Fc: 130bpm, Tax: $36,3^{\circ} \mathrm{C}$. Abdome globoso, ruídos hidroaéreos positivos, doloroso à palpação superficial e profunda, à palpação macicez difusa, esplenomegalia à $8 \mathrm{~cm}$ do rebordo costal esquerdo, sem sinal de irritação peritoneal.

Foram solicitados radiografia de tórax, hemograma completo, sumário de urina. Raio X de tórax e sumário de urina sem alterações. Hemograma revelou: hemácias 3,8 milhões; hemoglobina $8,8 \mathrm{~g} / \mathrm{dL}$; hematócrito $26 \%$; leucócitos globais $3900 \mathrm{~mm}^{3}$; plaquetas $145.000 \mathrm{~mm}^{3}$.

Optou-se por internação da paciente para investigação da hipótese diagnóstica de Leishmaniose visceral. Solicitouse teste rápido para LV, eletrocardiograma, hemograma, ureia, creatinina, velocidade de hemossedimentação (VHS), bilirrubina total e frações, TGO, TGP, amilase, albumina, proteínas totais e frações,

O teste rápido confirmou a hipótese diagnóstica previamente estabelecida, eletrocardiograma sem alterações e os novos exames laboratoriais mostraram: Hemácias: 3,6 milhões, hemoglobina: 9,0 g/dL, Hematócrito: 27\%; leucócitos globais: $4100 \mathrm{~mm}^{3}$; Plaquetas $150.000 \mathrm{~mm}^{3}$; ureia: $17 \mathrm{mg} / \mathrm{dL}$; creatinina: $0,6 \mathrm{mg} / \mathrm{dL}$; VHS: $55 \mathrm{~mm}$; bilirrubina total: $0,3 \mathrm{mg} / \mathrm{dL}$; bilirrubina indireta: $0,2 \mathrm{mg} / \mathrm{dL}$; bilirrubina direta: $0,1 \mathrm{mg} / \mathrm{dL}$; amilase: $153 \mathrm{mg} / \mathrm{dL}$; TGO:27 U/mL; TGP $15 \mathrm{U} / \mathrm{mL}$; Proteínas totais: 5,15; Albumina: 3,0 $\mathrm{mg} / \mathrm{dL}$; Globulina: $2,15 \mathrm{mg} / \mathrm{dL}$.

Paciente admitida em enfermaria de Pediatria foi iniciado Glucantime $20 \mathrm{mg} / \mathrm{kg} / \mathrm{dose}$. Abdome globoso, ruídos hidroaéreos positivos, refere desconforto à palpação abdominal profunda, à palpação maciez difusa, esplenomegalia à $6 \mathrm{~cm}$ do rebordo costal esquerdo, sem sinal de irritação peritoneal. Paciente evoluiu com melhora do estado geral, após início do tratamento, mantendo-se afebril. Foi completado o cartão vacinal da paciente.

\section{DISCUSSÃO}

A LV é uma doença parasitária potencialmente fatal, causada por várias espécies de protozoários intracelulares do gênero Leishmania. Indivíduos infectados pelo protozoário, podem desenvolver um quadro clínico de maior gravidade, caracterizado por febre prolongada, esplenomegalia, perda de peso, hepatomegalia e pancitopenia ${ }^{14,15}$.

A doença apresenta alta letalidade, podendo ultrapassar a taxa de $90 \%$, caso o tratamento medicamentoso não seja instituído. A LV é endêmica em mais de 60 países, com 500000 novos pacientes a cada ano em todo o mundo ${ }^{14,16}$

Paracatu, uma cidade localizada no noroeste do estado de Minas Gerais, possui uma população de 84.718 habitantes, distribuídos em uma área de 8.230 $\mathrm{km}^{2}$. Na zona rural residem $13 \%$ da população. A região é relativamente seca, possui relevo ondulado, plano e montanhoso; a extração de minérios e a agropecuária são as bases da economia da cidade. A cidade possui no total 43 estabelecimentos de saúde, 28 municipais e 15 privadas ${ }^{17}$.

O município de Paracatu, Minas Gerais, é uma área de intensa transmissão de LV, de acordo com o Ministério da Saúde. Dados do Sistema de Informação de Agravos de Notificação (SINAN), anualmente, no estado de Minas Gerais, notificam-se em média 445 casos confirmados de LV, num levantamento entre 2001 a $2010^{18}$.

A LV era uma doença comum em ambientes rurais; todavia, com o passar do tempo, ocorreu a urbanização da doença. A ocorrência de casos de LV na área urbana se deve a vários motivos, entre eles o desmatamento, que reduziu a oferta de alimentos para o mosquito transmissor, de modo que o homem e o cão se tornaram alternativas para obter alimento. Além disso, a ocupação urbana de forma desordenada, resultou em condições precárias de moradia e vida ${ }^{19,20}$. A cidade de Paracatu, Minas Gerais, é um bom exemplo da urbanização da LV, devido à ampla distribuição da doença na sua área urbana.

A doença é transmitida através da picada de fêmeas de flebotomíneos haematófagos do gênero Phlebotomus no Velho Mundo e Lutzomyia no Novo Mundo. O cão é o principal reservatório da doença ${ }^{10}$.

As manifestações típicas da LV incluem febre, perda ponderal, hepatoesplenomegalia, pancitopenia. Sintomas resultantes da replicação das formas amastigotas de Leishmania nos macrófagos do fígado, baço e medula óssea $^{21}$. No caso apresentado, a paciente apresentava todos os sintomas.

A maioria das pessoas diagnosticadas com LV apresentam infecções assintomáticas ou subclínicas e isto pode ser atribuído para o desenvolvimento de respostas, antiparasitários eficazes mediadas por células imunitárias. Todavia, pacientes podem apresentar quadro sintomático grave $^{22}$. Não estão completamente compreendidos os fatores que influenciam a suscetibilidade para a LV.

O estado nutricional pode influenciar a suscetibilidade à doença; a desnutrição afeta a imunidade inata, a imunidade mediada por células; a coinfecção com o vírus da imunodeficiência humana (HIV) leva a um quadro severo de imunodepressão, levando a manifestações clínicas graves ${ }^{23}$.

A resposta imunológica desencadeada pela Leishmania varia nos padrões Th1 e Th2. Tipicamente, a LV está associada a um padrão de resposta imune do tipo Th1, com citocinas pró-inflamatórias IL-1, IL-6, IL8, IL-12, IL-15, IFN-y e TNF. A LV quando associada a uma resposta imune do tipo Th2, caracterizada por níveis elevados de citocinas IL-4 e IL-13, sugere resultados clínicos desfavoráveis ${ }^{24}$. 
No hospedeiro vertebrado, as espécies de Leishmania causadoras de LV, tendem a se espalhar e alojar-se em reservatórios, como os macrófagos teciduais no baço, fígado e medula óssea ${ }^{25}$. A infecção persistente causada pela Leishmania está associada à mecanismos pelos quais o parasita consiga burlar, subverter o sistema imune do hospedeiro.

A leishmaniose é uma das hipóteses diagnósticas em pacientes que se apresentam com pancitopenia persistente, e uma história positiva de viagens a áreas endêmicas. É uma doença de notificação compulsória, no entanto, há subnotificação da doença pela falta de conhecimento da mesma por parte de alguns profissionais de saúde, e por muitas vezes os pacientes não procurarem o serviço de saúde.

O diagnóstico da LV pode ser feito por meio de técnicas sorológicas (ELISA, IMUNOFLUORESCÊNCIA INDIRETA) ou técnicas parasitológicas, evidenciando a presença do parasita nos tecidos do hospedeiro; vale ressaltar que são meios diagnósticos que nem sempre estão disponíveis nos serviços de saúde; devido seu custo, necessidade de laboratórios sofisticados, falta de precisão. Os testes rápidos de diagnóstico são fáceis de executar e estão disponíveis nos serviços de saúde 5 .

Os testes de diagnóstico rápido são dispositivos que não necessitam de profissional, nem laboratório altamente qualificados; os resultados podem ser lidos dentro de minutos a uma ou duas horas. Logo, se tornaram instrumento de grande importância no diagnóstico de LV.

A maioria dos testes de diagnóstico rápido funciona capturando um antígeno ou anticorpo sobre uma superfície sólida e então anexar moléculas para que se faça a detecção a olho nu. A técnica utilizada é a imunocromatográfica, com um formato de fluxo lateral ou vareta. Utiliza-se a proteína isolada a partir de Leishmania sp como antígeno. A forma recombinante da sequência 39-aminoácido de L. chagasi é o mais amplamente utilizado; este teste é conhecido como rK39. O rK39 TIC mostra alta sensibilidade e especificidade para o diagnóstico da leishmaniose visceral ${ }^{26,27}$.

O tratamento medicamentoso leishmanicida salva muitas vidas, porém o paciente pode apresentar recidiva do quadro clínico após tratamento, e até mesmo resistência à ação dos medicamentos durante o tratamento; tais características levam a uma dificuldade em tratar a LV. Além disso, as drogas disponíveis constituem arsenal terapêutico restrito; o antimonial pentavalente é uma droga muito tóxica, porém tem sido o pilar do tratamento da LV há décadas; atualmente, dá-se preferência à anfotericina $\mathrm{B}$ desoxicolato ou lipossomal.

Não há vacina disponível para a LV; logo, o tratamento medicamento é a única estratégia farmacológica para combater a Leishmania. As opções de tratamento disponíveis para a LV são limitadas.

Observa-se que no tratamento medicamentoso as drogas usadas apresentam muitos efeitos adversos; além disso há resistência por parte do protozoário às drogas utilizadas. São medicações de alto custo, além da necessidade de hospitalização para completar o tratamento. Todos esses fatores levam a um padrão insatisfatório na terapêutica instituída; reafirmando o conceito de doença negligenciada.

Conflito de interesse: Os autores declaram não haver conflito de interesse.

\section{REFERÊNCIAS}

1. Maciel DB, SILVA TAM, GOMES LI, et al. Infection with Leishmania (Leishmania) infantum of 0 to 18-month-old children living in a visceral leishmaniasis-endemic area in Brazil. Am J Trop Med Hyg. 2014;91(2):329-35. doi: 10.4269/ ajtmh.13-0418.

2. Alvar J, Velez ID, Bern C, Herrero M, Desjeux P, Cano J, Jannin J, den Boer M. Team WHOLC Leishmaniasis worldwide and global estimates of its incidence. PLoS ONE. 2012;7:e35671. http://dx.doi.org/10.1371/journal. pone. 0035671 .

3. Brasil. Ministério da Saúde, Secretaria de Vigilância em Saúde, Departamento de Vigilância Epidemiológica. Guia de vigilância epidemiológica. Brasília; 2009. p.1-816.

4. Brasil. Ministério da Saúde, Fundação Secretaria de Vigilância em Saúde, Departamento de Vigilância Epidemiológica. Manual de vigilância e controle da leishmaniose visceral. Brasília; 2006. p.1-122.

5. Boelaert M, Verdonck K, Menten J, et al. Rapid tests for the diagnosis of visceral leishmaniasis in patients with suspected disease. Cochrane Database Systematic Rev. 2014;(6):1-119. doi: 10.1002/14651858.CD009135.pub2.

6. Boelaert M, Criel B, Leeuwenburg J, Van Damme W, Le Ray D, Van der Stuyft P. Visceral leishmaniasis control: a public health perspective. Trans R Soc Trop Med Hyg. 2000;94(5):465-71.

7. Queiroz MJA, Alves JGB, Correia JB. Leishmaniose visceral: características clínico-epidemiológicas em crianças de área endêmica. J Pediatria.2004;80(2)141-6.

8. Badaró R, Jones TC, Lourenço B. A prospective study of visceral leishmaniasis in an endemic area of Brazil. J Infect Dis. 1986;154:639-49.

9. Alves JGB. Calazar. In: Figueira F, Ferreira OS, Alves JGB. Pediatria - Instituto Materno infantil de Pernambuco. $2^{\mathrm{a}}$ ed. Rio de Janeiro: Medsi; 1996. p.320-27.

10. Monge-Maillo B, López-Vélez R. Therapeutic options for visceral leishmaniasis. Drugs. 2013;73(17):1863-88. doi:10.1007/s40265-013-0133-0. 
11. Alvar J, Croft S, Olliaro P. Chemotherapy in the treatment and control of leishmaniasis. Adv Parasitol. 2006;61:22374. http://dx.doi.org/10.1016/S0065-308X(05)61006-8.

12. Croft SL, Sundar S, Fairlamb AH. Drug resistance in leishmaniasis. Clin Microbiol Rev. 2006;19:111-26. doi: 10.1128/CMR.19.1.111-126.2006.

13. Alvar J, Aparicio P, Aseffa A, et al. The relationship between leishmaniasis and AIDS: the second 10 years. Clin Microbiol Rev. 2008;21:334-59. doi: 10.1128/CMR.00061-07.

14. World Health Organization. Control of the leishmaniasis. Geneve; 2010. (WHO Technical Report Series, 949). Available from: http://apps.who.int/iris/ bitstream/10665/44412/1/WHO_TRS 949 eng.pdf.

15. Guerin PJ, Olliaro P, Sundar S, Boelaert M, Croft SL, Desjeux P, Wasunna MK, Bryceson AD. Visceral leishmaniasis: current status of control, diagnosis, and treatment, and a proposed research and development agenda. Lancet Infect Dis. 2002;2(8):494-501. http://dx.doi. org/10.1016/S1473-3099(02)00347-X.

16. Fu Q, Li S-Z, Wu W-P, et al. Endemic characteristics of infantile visceral leishmaniasis in the People's Republic of China. Parasites Vectors. 2013;6:143. doi: 10.1186/17563305-6-143.

17. Instituto Brasileiro de Geografia e Estatística. Censo demográfico 2010 [citado em 2016 mar. 25]. Disponível em: http://www.ibge.gov.br/cidadesat/topwin-Dow.htm?1.

18. Oliveira EM, Pimenta AM. Perfil epidemiológico das pessoas portadoras de leishmaniose visceral no município de Paracatu - MG no período de 2007 a 2010. Rev Min Enferm. 2014;18(2):365-70. doi: http://www.dx.doi. org/10.5935/1415-2762.20140028.

19. Monteiro ÉM, Silva JCF, Costa RT, Costa DC, Barata RA, Paula EV, et al. Leishmaniose visceral: estudo de flebotomíneos e infecção canina em Montes Claros, Minas Gerais. Rev Soc Bras Med Trop. 2005;38(2):147-52. http:// dx.doi.org/10.1590/S0037-86822005000200004.
20. Costa CHN, Tapety CMM, Werneck GL. Controle da leishmaniose visceral em meio urbano: estudo de intervenção randomizado fatorial. Rev Soc Bras Med Trop. 2007;40(4):415-9. http://dx.doi.org/10.1590/S003786822007000400009 .

21. Monge-Maillo B, Norman FF, Cruz I, Alvar J, LópezVélez R. Visceral Leishmaniasis and HIV Coinfection in the Mediterranean Region. Valenzuela JG, editor. PLoS Neglect Trop Dis. 2014;8(8):e3021. doi: 10.1371/journal. pntd.0003021.

22. Faleiro RJ, Kumar R, Hafner LM, Engwerda CR. Immune Regulation during Chronic Visceral Leishmaniasis. Melby PC, ed. PLoS Neglect Trop Dis. 2014;8(7):e2914. doi: 10.1371/journal.pntd.0002914.

23. Ostyn B, Gidwani K, Khanal B, Picado A, Chappuis $\mathrm{F}$, et al. Incidence of Symptomatic and Asymptomatic Leishmania donovani infections in High-Endemic Foci in India and Nepal: a prospective study. PLoS Negl Trop Dis. 2011;5:e1284. http://dx.doi.org/10.1371/journal. pntd.0001284

24. Ansari N, Saluja S, Salotra P. Elevated levels of interferon- $\gamma$, interleukin-10, and interleukin-6 during active disease in Indian kala azar. Clin Immunol. 2006;119:339-45. http:// dx.doi.org/10.1016/j.clim.2006.01.017

25. Maran N, Gomes OS, de Lima LF, Freitas EO, de Lima $\mathrm{CG}$, Morrot A. Host resistance to visceral leishmaniasis: prevalence and prevention. Expert Rev Anti-infective Ther. 2016:14:4. doi: 10.1586/14787210.2016.1160779.

26. Cunningham J, Hasker E, Das P, El Safi S, Goto H, Mondal $\mathrm{D}$, et al. A global comparative evaluation of commercial immunochromatographic rapid diagnostic tests for visceral leishmaniasis. Clin Infect Dis. 2012;55(10):1312-9. doi: 10.1093/cid/cis716.

27. World Health Organization. Control of the leishmaniasis: report of a meeting of the WHO Expert Committee on the Control of Leishmaniases. Geneva, 22-26 March 2010. Geneve; 2010. (WHO technical report series). 